\title{
Risk and Insurability of Storm Damages to Residential Buildings in Austria
}

\author{
Franz Prettenthaler ${ }^{\mathrm{a}, \mathrm{b}}$, Hansjörg Albrecher ${ }^{\mathrm{c}}$, Judith Köberl ${ }^{\mathrm{a}}$ and \\ Dominik Kortschak ${ }^{\mathrm{c}, \mathrm{d}, \mathrm{e}}$ \\ ${ }^{a}$ POLICIES - Centre for Economic and Innovation Research, JOANNEUM RESEARCH \\ Forschungsgesellschaft m.b.H., Leonhardstraße 59, Graz 8010, Austria. \\ E-mails: franz.prettenthaler@joanneum.at; judith.koeberl@joanneum.at \\ ${ }^{b}$ Wegener Center for Climate and Global Change, University of Graz, Austria. \\ ${ }^{c}$ Université de Lausanne, Quartier UNIL-Dorigny, Bâtiment Extranef, 1015 Lausanne. \\ E-mail: Hansjoerg.Albrecher@unil.ch \\ ${ }^{\mathrm{d}}$ Université de Lyon, F-69622, Lyon, France. \\ e'Université Lyon 1, Laboratoire SAF, EA 2429, Institut de Science Financiére et d'Assurances, 50 Avenue \\ Tony Garnier, F-69007 Lyon, France. \\ E-mail: kortschakdominik@gmail.com
}

This paper develops a stochastic model to assess storm risk in Austria, which relates wind speed and actual losses. By virtue of a building-stock-value-weighted wind index, we use suitably normalised historical loss data of residential buildings over 12 years and corresponding wind speed data to calibrate the model. Subsequently, additional wind speed data is used to generate further scenarios and to obtain loss curves for storm risk that give rise to storm insurance loss quantiles and corresponding solvency capital requirements both on the aggregate and on the regional level. We also investigate the diversification effect across regions and use tools from extreme value theory to assess the insurability of storm risk in Austria in general.

The Geneva Papers (2012) 37, 340-364. doi:10.1057/gpp.2012.15

Keywords: storm damage; residential buildings; extreme value statistics; spatial dependence model; insurability; Austria

\section{Introduction}

Directive 2009/138/EC ${ }^{1}$ of the European Parliament and of the Council of 25 November 2009 (Solvency II) is the basis of a broad regulatory project for the supervision of (re)insurance undertakings that wants to strengthen the risk-based approach to this supervision and is reshaping risk management throughout Europe. The directive clearly sets out how such an enterprise needs to calculate the prescribed solvency capital requirement from the various risks it is exposed to, also for non-life catastrophic risk. When calculating its Solvency II conform Value-at-Risk subject to a confidence level of 99.5 per cent over a 1-year period, the individual insurance company has the choice to either adopt the standard formula or develop, certificate, implement and maintain permanently an internal model. For small to medium sized

\footnotetext{
${ }^{1}$ Directive 2009/138/EC.
} 


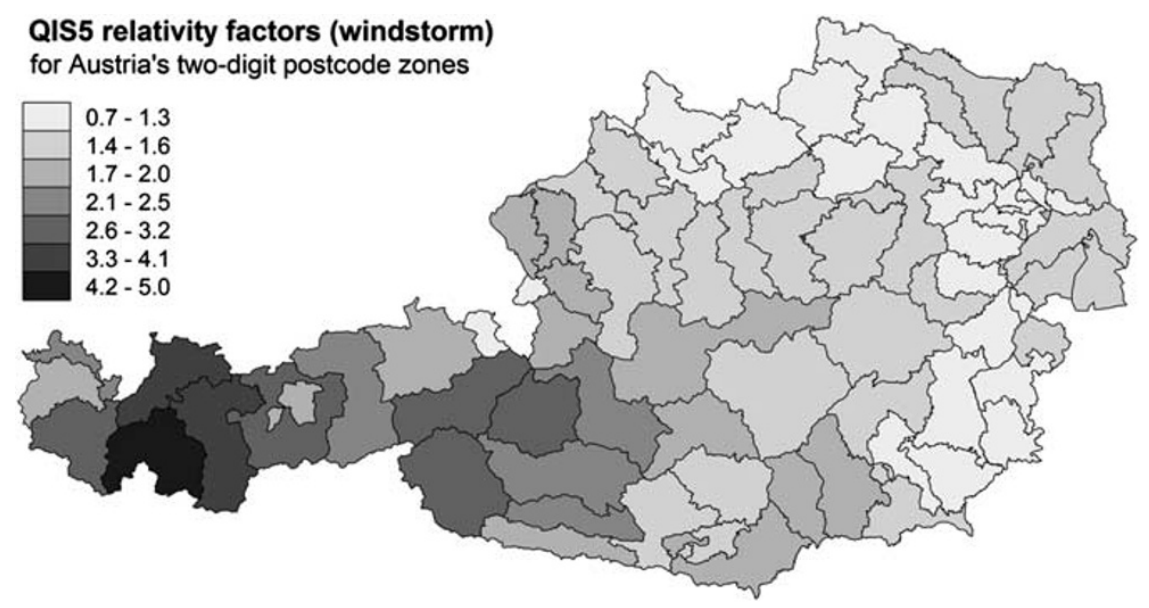

Figure 1. QIS5 relativity factors (windstorm) for Austria's CRESTA zones (European Commission (2010b); worksheet WS_CRESTA_AT).

insurance companies the high fixed costs of setting up a full internal model covering all risks certainly would mean a competitive disadvantage, so this is why adopting the standard formula seems to be an interesting option. Thus all technical specifications for the implementation of the standard formula are followed with strong interest within the industry. However, when the relativity factors for the Austrian Catastrophe Risk Evaluating and Standardizing Target Accumulations (CRESTA) zones for windstorm were published in the course of the 5th Quantitative Impact Study on Solvency II (QIS5) (see Figure 1), they came as a big surprise, because they seemed to contradict the damage experience of the last decades that, according to loss data provided by the Austrian Association of Insurers (VVO), seemed to concentrate in Upper Austria - at least for residential buildings (see the topmost plot in Figure 2). Since the underlying modelling assumptions of QIS5 have not been published, we only can reckon that the strong loading factor for the western part of Austria (especially Tyrol) illustrated in Figure 1, may come from an unspecific choice of weather stations and a resulting overly simplistic interpretation of wind speed that certainly is highest on the highest (and sparsely populated) mountains, but does not necessarily match with actual damage experience in the rather protected valleys, where most of the population is located. However, this observation must remain speculation as long as neither QIS5 modellers nor developers of commercial software, that show the same obvious bias, reveal their modelling details or supporting damage data.

This confusion on the regional distribution of storm risk, with potentially strong economic impacts for individual Austrian insurance companies, hits the industry in a situation that already contains some uneasiness concerning storm risk. Over the last years there has been an increasing concern about the insurability of losses due to storms in view of the frequent storm events and large corresponding losses in Austria. In the course of this discussion, the Austrian Association of Insurers (VVO) collected and provided insurance loss data on storm events over a 12-year period, aggregated across different companies, but for each storm event specified with a resolution of 


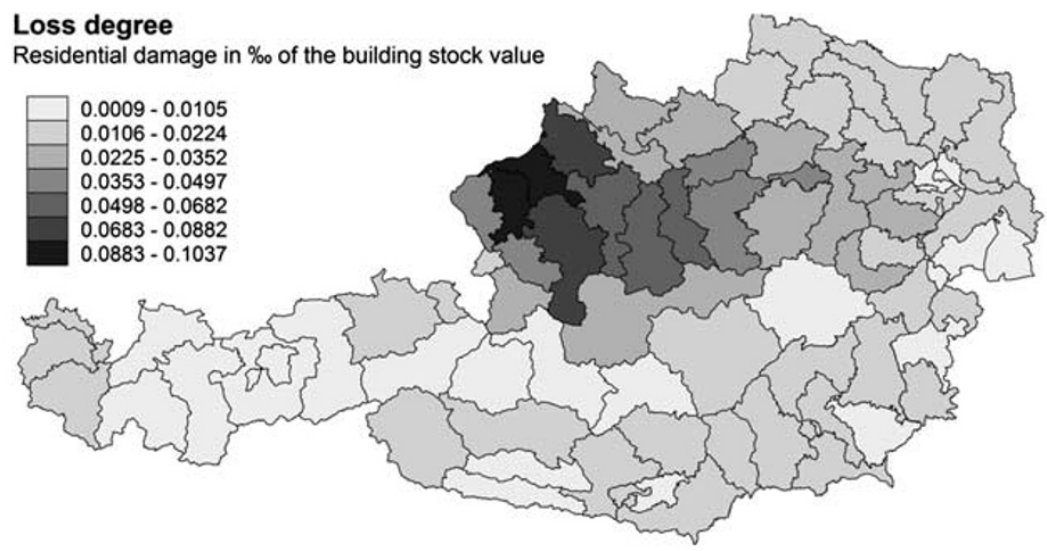

Wind index

Building-stock-value-weighted maximum wind speed
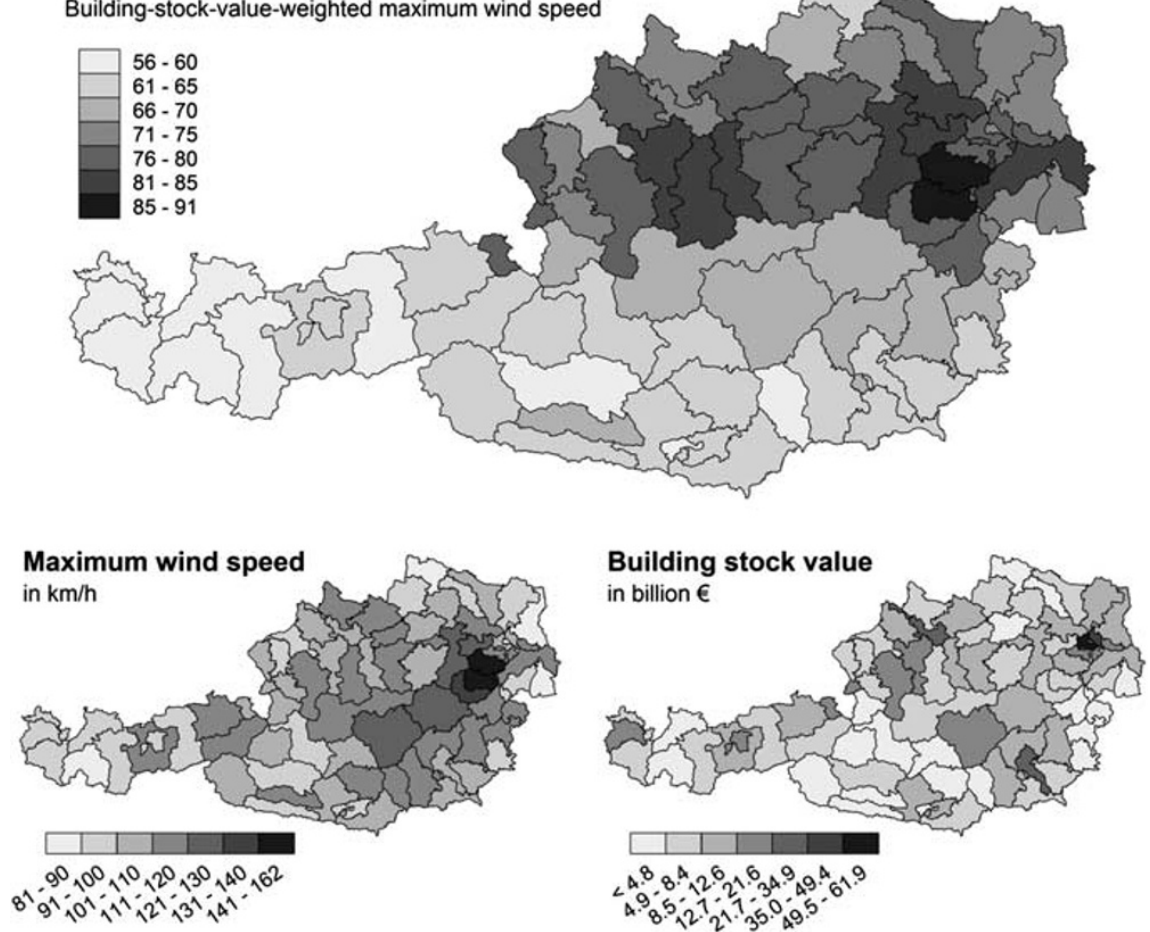

Figure 2. Loss degree, wind index, maximum wind speed and building stock value for an average storm event of the period 1998-2009 (with 36 storm events being considered).

two-digit postcode regions, which correspond to the CRESTA zones. The aim of this paper is to use this data set for setting up and calibrating a stochastic model for storm risk in Austria. For that purpose, we use detailed data on maximum wind speeds during storm events between 1998 and 2009 that caused insured losses of 
at least $€ 3$ million throughout Austria (measured in $€ 2009$ ) and create a buildingstock-value-weighted wind index for each region and each storm (i.e. on a finer scale within each region the wind speed is weighted according to the number of buildings in this particular area), which gives a more representative description of the wind speed in terms of loss potential. For practical reasons we restrict the analysis to residential buildings, which is anyway the most important part of the storm risk assessment. ${ }^{2}$ The historical loss data of residential buildings are normalised both with respect to inflation and with respect to the building stock value in the area, as the same wind speed will have a different impact on the same region at different times due to those two factors.

After discussing the available data in the following section, we use the (suitably normalised) aggregate loss of each storm and study the insurability of storm risk in Austria on a rough level in the section after that, using techniques from extreme value theory (EVT). In the subsequent section we then formulate a more refined stochastic model that relates wind speed and loss amounts. We use the available loss experience for calibrating the model and then use additional wind field data during additional storms in the past (back to 1974) to create further scenarios, for which we can run simulations of the model (section "Enlarging the set of available scenarios"). After that we discuss a procedure to extend the per-event analysis to annual estimates. This results in loss curves for storm risk in Austria both on the aggregate and on the regional level, which we discuss in the penultimate section, together with implications of the model for quantifying the diversification effect that emerges when all provinces are collected in one pool, an issue that is particularly relevant for insurance companies when designing optimal strategies to cope with storm risk. We state these results also in terms of resulting capital requirements in the Solvency II framework for insurance regulation in the European Union. The last section concludes.

\section{Available data}

The Austrian Association of Insurers (VVO) provided a list of storm events for the period 1974 to 2009 by means of various sources of observation, resulting in a total number of 96 storm events for this period (see Table 1). For each of these storm events, reanalysis data on maximum $10 \mathrm{~m}$ wind speeds (i.e. the maximum of hourly wind speeds per storm event) were provided by the Central Institute for Meteorology and Geodynamics (ZAMG) through their Integrated Nowcasting through Comprehensive Analysis (INCA) System $^{3}$ on a $1 \times 1 \mathrm{~km}$ horizontal resolution, which corresponds to about 85,000 grid cells. In addition, monetary damage data (insurance loss data $)^{4}$ - adjusted for inflation and market shares - were provided by the VVO on

\footnotetext{
${ }^{2}$ See for example Heneka and Ruck (2008).

${ }^{3}$ INCA represents a high-resolution weather analysis (and prediction) model of the ZAMG. Gridded wind speed data results from an algorithmic combination of model data and observational data and is available on a spatial resolution of $1 \mathrm{~km}$ and a temporal resolution of $1 \mathrm{~h}$. For more detailed information on INCA and its validation see Haiden et al. (2011).

${ }^{4}$ Note that, although this damage data should only contain storm related losses, indirect storm losses associated with rain and hail may be included as well.
} 
Table 1 List of storm events

\begin{tabular}{|c|c|c|c|c|}
\hline$\#$ & Name & Start date & End date & $I L^{\mathrm{a}}$ [in $\left.€ 2009\right]$ \\
\hline 1 & - & 17.01.1974 & 17.01.1974 & - \\
\hline 2 & - & 29.12 .1974 & 29.12 .1974 & - \\
\hline 3 & Capella & 03.01 .1976 & 03.01 .1976 & - \\
\hline 4 & - & 31.12 .1977 & 31.12 .1977 & - \\
\hline 5 & - & 03.01 .1981 & 03.01 .1981 & - \\
\hline 6 & - & 04.02 .1981 & 04.02 .1981 & - \\
\hline 7 & - & 26.04 .1981 & 26.04 .1981 & - \\
\hline 8 & - & 11.10 .1981 & 11.10 .1981 & - \\
\hline 9 & - & 09.10 .1982 & 09.10 .1982 & - \\
\hline 10 & - & 10.11 .1982 & 10.11 .1982 & - \\
\hline 11 & - & 28.12 .1983 & 28.12 .1983 & - \\
\hline 12 & - & 24.11 .1984 & 24.11 .1984 & - \\
\hline 13 & - & 18.12 .1985 & 18.12 .1985 & - \\
\hline 14 & - & 31.01 .1986 & 31.01 .1986 & - \\
\hline 15 & - & 19.12 .1986 & 19.12 .1986 & - \\
\hline 16 & - & 05.04 .1987 & 05.04.1987 & - \\
\hline 17 & - & 14.02 .1990 & 14.02 .1990 & - \\
\hline 18 & Vivian & 26.02 .1990 & 27.02 .1990 & - \\
\hline 19 & Wiebke & 28.02 .1990 & 01.03 .1990 & - \\
\hline 20 & - & 18.11 .1990 & 18.11 .1990 & - \\
\hline 21 & - & 21.03 .1992 & 22.03 .1992 & - \\
\hline 22 & - & 24.01 .1993 & 24.01 .1993 & - \\
\hline 23 & - & 18.07.1993 & 18.07.1993 & - \\
\hline 24 & - & 08.12 .1993 & 08.12 .1993 & - \\
\hline 25 & - & 06.01 .1994 & 06.01 .1994 & - \\
\hline 26 & Lore & 28.01 .1994 & 29.01 .1994 & - \\
\hline 27 & - & 13.03. 1994 & 14.03 .1994 & - \\
\hline 28 & Wilma & 26.01.1995 & 27.01 .1995 & - \\
\hline 29 & - & 12.06 .1995 & 12.06 .1995 & - \\
\hline 30 & - & 06.11 .1995 & 07.11 .1995 & - \\
\hline 31 & - & 11.11.1996 & 12.11.1996 & - \\
\hline 32 & - & 13.02.1997 & 13.02.1997 & - \\
\hline 33 & - & 05.04 .1997 & 05.04.1997 & - \\
\hline 34 & - & 07.04.1997 & 08.04.1997 & - \\
\hline 35 & - & 11.04.1997 & 13.04.1997 & - \\
\hline 36 & - & 02.01 .1998 & 02.01 .1998 & - \\
\hline 37 & - & 05.01 .1998 & 06.01 .1998 & - \\
\hline 38 & - & 07.07.1998 & 07.07.1998 & $3,339,571$ \\
\hline 39 & - & 27.07.1998 & 27.07.1998 & - \\
\hline 40 & - & 31.07 .1998 & 01.08.1998 & $3,573,821$ \\
\hline 41 & - & 28.10 .1998 & 29.10 .1998 & - \\
\hline 42 & - & 01.11 .1998 & 01.11 .1998 & - \\
\hline 43 & Lara & 04.02.1999 & 05.02.1999 & $4,365,383$ \\
\hline 44 & - & 01.06.1999 & 04.06.1999 & $13,130,338$ \\
\hline 45 & Lothar & 26.12.1999 & 26.12.1999 & $21,979,805$ \\
\hline 46 & - & 17.01.2000 & 21.01 .2000 & $8,596,491$ \\
\hline 47 & - & 04.07 .2000 & 04.07 .2000 & $25,657,578$ \\
\hline 48 & - & 09.03 .2000 & 09.03.2000 & - \\
\hline 49 & - & 07.07 .2000 & 07.07.2000 & $3,187,199$ \\
\hline
\end{tabular}


Table 1 (continued)

\begin{tabular}{|c|c|c|c|c|}
\hline$\#$ & Name & Start date & End date & $I L^{\mathrm{a}}$ [in $\left.€ 2009\right]$ \\
\hline 50 & - & 06.11 .2000 & 06.11 .2000 & - \\
\hline 51 & Emma & 21.02 .2001 & 21.02 .2001 & - \\
\hline 52 & - & 19.03 .2001 & 19.03.2001 & - \\
\hline 53 & - & 07.07 .2001 & 07.07.2001 & $5,289,547$ \\
\hline 54 & - & 02.01 .2002 & 02.01 .2002 & - \\
\hline 55 & - & 23.06 .2002 & 24.06 .2002 & $5,225,198$ \\
\hline 56 & - & 10.07 .2002 & 10.07 .2002 & $2,152,088$ \\
\hline 57 & - & 11.08 .2002 & 13.08 .2002 & $5,264,797$ \\
\hline 58 & Jeanette & 27.10 .2002 & 28.10 .2002 & $13,710,943$ \\
\hline 59 & - & 16.11.2002 & 17.11.2002 & $9,252,369$ \\
\hline 60 & Calvann & 01.01 .2003 & 03.01 .2003 & $3,881,060$ \\
\hline 61 & - & 06.04 .2003 & 07.04 .2003 & - \\
\hline 62 & - & 13.05 .2003 & 13.05 .2003 & $2,679,134$ \\
\hline 63 & - & 14.06 .2003 & 15.06.2003 & - \\
\hline 64 & - & 21.07 .2003 & 21.07.2003 & - \\
\hline 65 & - & 18.08 .2003 & 18.08.2003 & - \\
\hline 66 & - & 28.08 .2003 & 30.08 .2003 & $3,950,117$ \\
\hline 67 & - & 07.10 .2003 & 07.10 .2003 & - \\
\hline 68 & Gerda-Hanne & 12.01 .2004 & 15.01 .2004 & $3,915,547$ \\
\hline 69 & Ursula & 19.01.2004 & 19.01 .2004 & - \\
\hline 70 & - & 08.02 .2004 & 08.02 .2004 & - \\
\hline 71 & - & 06.07 .2004 & 06.07 .2004 & - \\
\hline 72 & - & 12.08 .2004 & 13.08 .2004 & $3,611,801$ \\
\hline 73 & - & 18.11.2004 & 20.11 .2004 & $14,126,622$ \\
\hline 74 & - & 30.05 .2005 & 30.05 .2005 & $2,606,848$ \\
\hline 75 & Dorian & 18.07.2005 & 18.07.2005 & - \\
\hline 76 & - & 15.12 .2005 & 16.12 .2005 & - \\
\hline 77 & - & 01.01 .2006 & 07.01 .2006 & $7,741,211$ \\
\hline 78 & - & 27.06 .2006 & 30.06 .2006 & $10,486,044$ \\
\hline 79 & - & 29.08 .2006 & 29.08 .2006 & 116,958 \\
\hline 80 & - & 05.11 .2006 & 05.11 .2006 & - \\
\hline 81 & Franz & 11.01 .2007 & 13.01.2007 & $4,408,424$ \\
\hline 82 & Kyrill & 18.01 .2007 & 20.01.2007 & $161,831,725$ \\
\hline 83 & Olli & 28.01 .2007 & 29.01 .2007 & $9,289,850$ \\
\hline 84 & - & 20.06 .2007 & 22.06 .2007 & $19,820,640$ \\
\hline 85 & Klaus & 11.11 .2007 & 11.11.2007 & - \\
\hline 86 & Paula & 26.01 .2008 & 28.01.2008 & $63,877,821$ \\
\hline 87 & Emma & 01.03 .2008 & 03.03.2008 & $111,407,420$ \\
\hline 88 & - & 23.06 .2008 & 27.06 .2008 & $13,289,295$ \\
\hline 89 & - & 12.07.2008 & 12.07.2008 & $3,717,986$ \\
\hline 90 & - & 14.08 .2008 & 15.08.2008 & $4,464,130$ \\
\hline 91 & - & 22.08 .2008 & 22.08 .2008 & $8,628,437$ \\
\hline 92 & Felix & 26.05.2009 & 27.05.2009 & $4,466,481$ \\
\hline 93 & - & 24.01 .2009 & 24.01.2009 & - \\
\hline 94 & - & 16.06 .2009 & 16.06.2009 & $2,858,010$ \\
\hline 95 & - & 06.07 .2009 & 07.07.2009 & $4,674,891$ \\
\hline 96 & Wolfgang & 23.07.2009 & 24.07.2009 & $115,521,121$ \\
\hline
\end{tabular}

${ }^{\mathrm{a}} \mathrm{IL}=$ Insured losses to residential buildings. 
346

a two-digit postal code basis for those storm events that occurred between 1998 and 2009 and caused a total insured damage of at least $€ 3$ million (measured in $€ 2009$ ) throughout Austria, which amounts to 36 storm events. ${ }^{5}$ The term "total damage" in this case comprises damages to residential and industrial buildings and their contents, damages to machines and damages to goods in transit, which represent the lines of business covered by the VVO data. However, for the analysis in this paper we only consider damages to residential buildings (excluding contents) ${ }^{6}$ which account for 83 per cent of the total damages outlined by the VVO data. Correspondingly, roughly speaking we use damage data for those storm events that caused damages to residential buildings (excluding contents) of at least $\sim € 2.5$ million.

In addition to this data provided on past storm events, we developed a spatially highly resolved, inflation-adjusted annual time series of the building stock value in a three-step procedure by using GIS-methods. On the basis of Statistic Austria's buildings and dwellings censuses of 1971, 1981, 1991 and 2001, in a first step an annual time series of the residential floor space was generated on a spatial resolution of $250 \times 250 \mathrm{~m}$ for the period 1974-2009. Subsequently, the building stock of each grid cell was assessed by its new construction value (excluding contents) currently in force, whereas the final step of the procedure involved the adjustment of the resulting time series for inflation. The resulting time series of the building stock value was used on the one hand to aggregate the data on maximum wind speeds from the $1 \times 1 \mathrm{~km}$ resolution to two-digit postcode level by forming building-stock-value-weighted averages - referred to as "wind index" in the following - and on the other hand to normalise the data on storm damages by expressing it in per mille of the building stock value.

Figure 2 illustrates the spatial distribution of loss degree, wind index, maximum wind speed and building stock value for an average storm event of the period 1998-2009. With respect to the spatial distribution of the loss degree the illustration shows that on average the two-digit postcode regions in "Upper Austria" faced the highest normalised damages within the observation period. On the aggregate level, Figure 3 depicts the normalised losses per storm event. The largest damage was caused by storm Kyrill (18-20 January 2007) with about €162 million (which amounts to a loss degree of 0.199 per thousand).

\section{EVT methodology for the aggregate losses}

Using the numbers depicted in Figure 3, one can study the distribution of the (normalised) aggregate storm loss. Whereas the analysis for the region around the median of the distribution can be based on the empirical distribution function or some suitably general parametric family, it is the upper tail of the distribution that is

\footnotetext{
${ }^{5}$ Two of the original 38 storm events with damage data represented some kind of outliers and were removed from the database used for analyses. Concretely, the storm event of 29 August 2006 led to residential damages of $€ 117,000$, which is far below the limit to be included, whereas storm "Wolfgang", which occurred on 23-24 July 2009, shows overly excessive damages given the observed wind speeds, which might be attributed to tainted separation of flood and hail damages accompanying the storm event.

${ }^{6}$ The fifth column in Table 1 outlines the damages to residential buildings throughout Austria per storm event.
} 


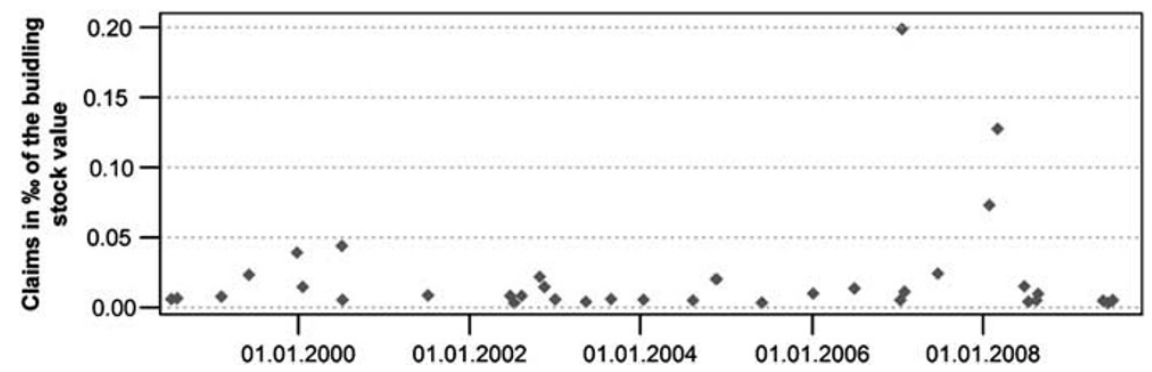

Figure 3. Time series of the 36 (normalised) aggregate claims per storm (1998-2009).

of prime importance for assessing the risk associated with storm events. However, for such extreme events there are only a few data points available and we have to resort to techniques from EVT. For a general overview of techniques and methods in that field we refer to de Haan and Ferreira, ${ }^{7}$ Reiss and Thomas, ${ }^{8}$ or Embrechts et al. ${ }^{9}$ The EVT approach takes a sophisticated compromise between choosing only few data points that can be assigned to extreme events and at the same time gathering sufficiently many data points to infer reliable conclusions on the excess distribution of new records, allowing estimation of large quantiles of the distribution. In order to apply the classical tools of EVT, we will assume that our observations (the aggregate losses of the individual storms) are independent and identically distributed random variables $X_{1}, \ldots, X_{n}$, an assumption that seems acceptable (however, one should keep in mind that both independence and stationarity are strong assumptions so that the results should rather be seen as rough estimates). For the tail of its distribution, from EVT it seems natural to look for a power-type decay, namely $P(X>x) \sim c \cdot x^{-\alpha}$ for $x \rightarrow \infty$, where the constant $\alpha>0$ is the crucial quantity and $c>0$ is another constant. Let $X_{1, n} \leqslant \cdots \leqslant X_{n, n}$ denote the corresponding order statistic. Then the so-called Hill estimator for $\alpha$ is defined through:

$$
H_{k, n}=\left(\frac{1}{k} \sum_{j=1}^{k} \log \left[X_{n-j+1, n}\right]-\log \left[X_{n-k, n}\right]\right)^{-1}
$$

where $k$ is an index that needs to be chosen suitably, and for an increasing number of observations $n \rightarrow \infty$, the estimator $H_{k, n}$ is known to converge to the actual value of $\alpha{ }^{10}$ Figure 4 gives the corresponding Hill plot. The choice of $k$ for finite sample sizes is a difficult compromise between bias and variance, and usually one looks for a horizontal section in the plot for not too large $k$ to read off the estimate for $\alpha$. In the present situation one would deduce the estimate $\hat{\alpha} \approx 1.1$ (the dashed lines in the plot

\footnotetext{
${ }^{7}$ de Haan and Ferreira (2006).

${ }^{8}$ Reiss and Thomas (2007).

${ }^{9}$ Embrechts et al. (1997).

${ }^{10}$ Beirlant et al. (2004).
} 


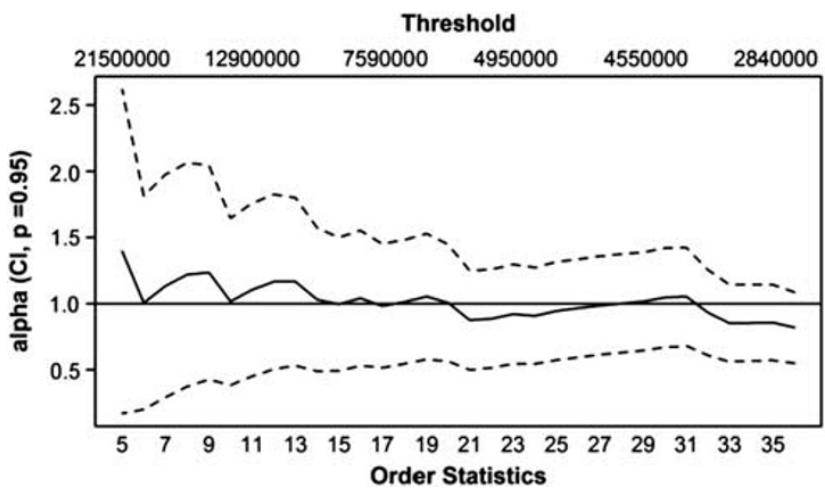

Figure 4. Hill plot of the normalised aggregate claims.

correspond to the 95 per cent confidence interval). An alternative is the so-called moment estimator:

$$
\begin{aligned}
& M_{k, n}=\left(H_{k, n}\right)^{-1}+1-\frac{1}{2}\left(1-\frac{H_{k, n}^{-2}}{H_{k, n}^{(2)}}\right)^{2}, \quad \text { with } \\
& H_{k, n}^{(2)}=\frac{1}{k} \sum_{j=1}^{k}\left(\log \left[X_{n-j+1, n}\right]-\log \left[X_{n-k, n}\right]\right)^{2},
\end{aligned}
$$

where $k$ is again to be chosen from the horizontal section in the plot. ${ }^{11}$ Figure 5 shows the moment plot for this data set, which depicts $1 / \alpha$ and would indicate a slightly larger estimate $\alpha \approx 1.25$. Since the Hill estimator usually has the smaller variance, ${ }^{6}$ one would conjecture the actual value of $\alpha$ in any case close to, but greater than 1 . This implies a finite expected value of the aggregate storm risk and hence insurability on the general level. An $\alpha$-value slightly above 1 for storm losses is also in accordance with studies for data in other countries. ${ }^{12}$

\section{A spatial model relating wind speed and damage}

We now want to set up a spatially refined (causal) stochastic model that relates the generated insurance loss in a region to the maximum wind speed of that region. ${ }^{13,14}$

${ }^{11}$ For large $n$ there are refined algorithms to identify $k$, see for example Draisma et al. (1999). Unfortunately our magnitude for $n$ is not sufficiently large for those algorithms.

${ }^{12}$ See for example Lescourret and Robert (2006).

${ }^{13}$ Storm loss models with high spatial resolutions can also be found for example in Heneka and Ruck (2008) or Donat et al. (2011). Both studies also give an overview on storm loss models found in the recent literature.

${ }^{14}$ In view of the limited amount of available data we have to make the compromise to use a model with only a few parameters. Naturally such a model will be — as any other model - a simplification that cannot capture all effects that determine the relation in reality, yet it takes into account crucial aspects in a way 


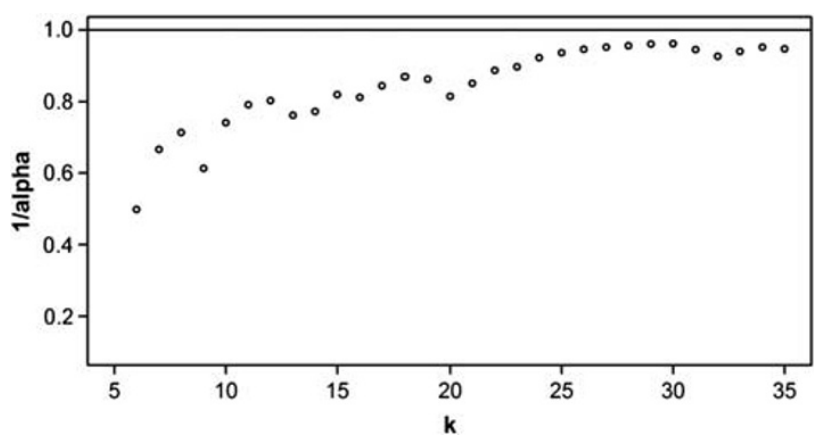

Figure 5. Moment plot of the normalised aggregate claims.

For the wind speed, we will use the building-stock-value-weighted wind index discussed above. In the literature one can find different approaches to model this relationship. For instance, in Klawa and Ulbrich, ${ }^{15}$ Pinto et al., ${ }^{16}$ or Donat et al. ${ }^{17}$ the loss damage is set proportional to the third power of the wind speed, which is motivated by the fact that wind energy is related to the cube of the wind speed. However, one should note that the relation between damage and energy is not necessarily linear. Another approach followed in Dorland et al. ${ }^{18}$ is to model the damage proportional to the exponent of the wind speed. Figure 6 shows a plot of wind speed against the logarithm of the induced insurance loss across all regions and storms. On the basis of this plot, we decided to use an exponential relationship. However, opposed to Dorland et al., ${ }^{18}$ who calibrated their model using data of the Netherlands, we will also take into account the topographic differences and the differences in the building structure between regions by introducing a region-specific correction (in Austria the characteristics among regions are more diverse than in the Netherlands). ${ }^{19}$

Further we assume that every storm event has its own characteristic such as duration, proportion of gusts, affected area in a region, but also the damage proneness of affected buildings, ${ }^{20}$ which-although not representing a physical characteristic of

we consider somewhat satisfactory for the present purpose, given the constraints on the data side. Since there is always some degree of arbitrariness in the choice of the model, the justification of using exactly this type of model has to remain on a vague level.

15 Klawa and Ulbrich (2003).

16 Pinto et al. (2007).

${ }^{17}$ Donat et al. (2011).

18 Dorland et al. (1999).

${ }^{19}$ Klawa and Ulbrich (2003) weigh the different areas according to the (somewhat arbitrary) 98 per cent quantile of daily maximum wind speeds, arguing that in more prone areas houses are better adapted against storms. Apart from the fact that we do not have the same data information available, in our approach we project this effect together with other regional effects in one region-specific (multiplicative) correction factor, which is calibrated with the available data. As any other approach, this is clearly a simplification of the real situation.

${ }^{20}$ For example after a long winter one could assume that more roofs are in a bad shape than at the beginning of the winter and hence a storm with the same wind field can lead to quite different losses. 


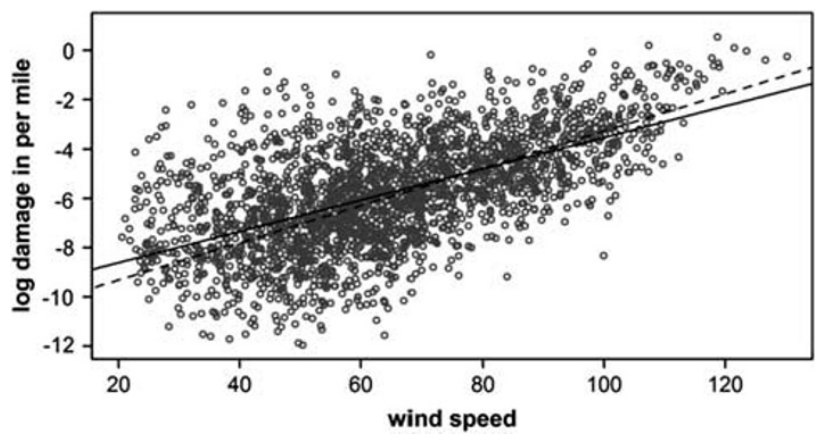

Figure 6. Wind speed (in $\mathrm{km} / \mathrm{h}$ ) against the logarithm of the induced loss degree across regions and storms inclusive least-squares regression line with (dashed line) and without (full line) weights.

the storm event itself - does affect its damage potential. We model this effect by a storm-specific correction factor. Denote by $X_{i, j}$ the logarithm of the damage of storm $i$ in region $j$ and with $W_{i, j}$ the corresponding wind index. For the expected loss of storm $i$ in region $j$ we now set:

$$
E\left[X_{i, j}\right]=s W_{i, j}+I^{L M}+I_{i}^{S}+I_{j}^{G} .
$$

That is, the slope $s$ is the same for all storms, but the intercept $I^{L M}+I_{i}^{S}+I_{j}^{G}$ depends on the storm (index $i$ ) as well as on the region (index $j$ ). Whereas the regionspecific factor $I_{j}^{G}$ is assumed deterministic, the storm-specific $I_{i}^{S}$ will be assumed to follow a normal distribution $N\left(\mu_{I}, \sigma_{I}^{2}\right)$, as later we will use further storm scenarios for which we need to sample from this distribution for the correction factor. Finally we assume that the deviation of the actual random variable $X_{i, j}$ from its mean follows a two-sided Gamma distribution $\Gamma(v, \beta)$ with density $f(x) \propto x^{v-1} e^{-\beta x}$. Altogether we hence postulate:

$$
X_{i, j} \sim N\left(s W_{i, j}+I_{j}^{G}+\mu_{I}, \sigma_{I}^{2}\right)+S \cdot \Gamma(v, \beta),
$$

where $P(S=1)=0.5=P(S=-1)$.

Regarding model calibration, two different methods are discussed below. To that end, the following comment is in order: as the model has to be a simplification of reality, one should not expect a perfect fit to the data. We are eventually interested in high quantiles of the loss distribution; hence the calibration procedure should reasonably emphasise the tail behaviour. In addition, the data quality is not ideal, and summer storm data is (partially) contaminated. Method 1 below is an ad hoc approach that incorporates some of these aspects. In Method 2, on the other hand, we implement a Bayesian approach, which cannot account for our focus on the tail behaviour, but may be interpreted as the statistically cleaner procedure. We implement both procedures, but tend to favour Method 1. 


\section{Method 1}

We have to estimate the following parameters: the slope $s$, the intercepts $I_{j}^{G}$ of the regions, the parameters $\mu_{I}$ and $\sigma_{I}^{2}$ for the intercepts of storms (the intercept $I^{L M}$ is redundant, as it can be incorporated into the other intercepts) and finally the parameters for the residuals $v$ and $\beta$. For the calibration of the model we only used storms for which the sum of the wind indices exceeds 3,500 (this is an average of $45.5 \mathrm{~km} / \mathrm{h}$, excluding four storm events; the reason is to avoid selection bias due to lower truncation of the data provided by the VVO). At first we estimate the parameter $s$ using a weighted least-squares method, where the weights for each storm are proportional to the difference between its largest and smallest wind index, and halving the weight for summer storms (which reportedly have a higher inaccuracy due to mixing with damage from accompanied floods in the provided database). This amounts to the estimate $\hat{s}=0.07549$ and $\hat{I}^{L M}=-10.82$. In Figure 6 we see the effect of the weights on the least-squares regression (full line: without weights; dashed line: with weights). Figure 7 depicts this difference for two specific storm events (i.e. in these two plots, each dot corresponds to the damage of a region during that storm). To estimate now the parameters $\mu_{I}$ and $\sigma_{I}$ for the intercept-correction $I_{i}^{S}$ of the storms, we computed the empirical means $\hat{I}_{i}^{S}=1 /\left(\#\left\{j \mid X_{i, j}>-\infty\right\}\right) \sum_{j \mid X_{i, j}>-\infty}\left(X_{i, j}-\hat{s} W_{i, j}-\hat{I}^{L M}\right)$ for every storm, from which (using the same weights as for the slope) we obtain the parameter estimates $\hat{\mu}_{I}=-0.0378$ and $\hat{\sigma}_{I}^{2}=0.669$. Figure 8 shows a probability plot for the fitted normal distribution. Next, for the intercept $I_{j}^{G}$ of the region $j$ we again use the empirical mean $\hat{I}_{j}^{G}=1 /\left(\#\left\{i \mid X_{i, j}>-\infty\right\}\right) \sum_{i \mid X_{i, j}>-\infty}\left(X_{i, j}-\hat{s} W_{i, j}-\hat{I}^{L M}\right)$ to determine the (deterministic) correction factors, see Table 2 and Figure 9 for the results.

Finally, to estimate the parameters $v$ and $\beta$ of the Gamma distribution of the residuals we only use the winter storm data. Since the residuals $R_{i, j}=X_{i, j}-\hat{s} W_{i, j}$ $\hat{I}_{i}^{S}-\hat{I}_{j}^{G}-\hat{I}^{L M}$ are naturally quite different across regions, yet the weighting should be commensurate with actual importance (in terms of absolute aggregate loss of that
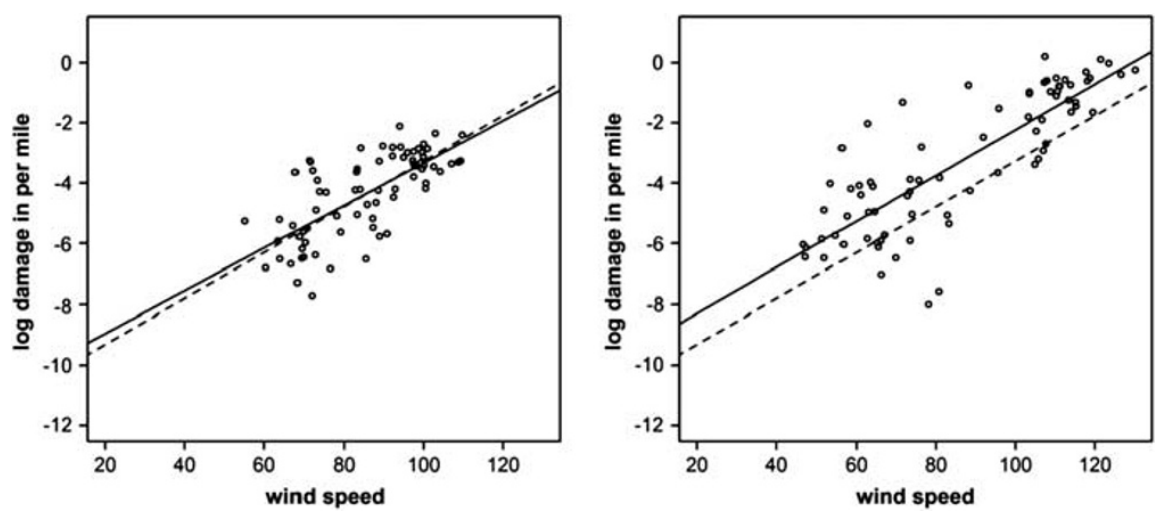

Figure 7. Linear relation between log damages and wind speed for the storms Jeannette of 27-28 October 2002 (left) and Kyrill of 18-21 January 2007 (right) (least-squares-fit without (full line) and with (dashed line) weights). 


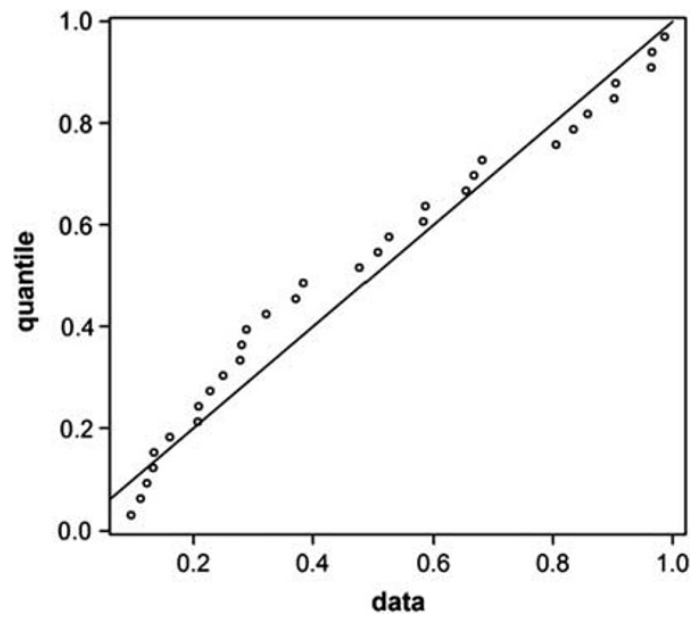

Figure 8. PP plot for the storm intercept and its estimated normal distribution.

Table 2 Correction factors for each two-digit postcode region

\begin{tabular}{lrlrlrrr}
\hline$P C$ & \multicolumn{1}{c}{$P C$} & \multicolumn{1}{c}{$I_{j}^{G}$} & \multicolumn{1}{c}{$P C$} & \multicolumn{1}{c}{$I_{j}^{G}$} & $P C$ & $I_{j}^{G}$ \\
\hline $10 \mathrm{xx}$ & -1.06 & $37 \mathrm{xx}$ & -1.09 & $56 \mathrm{xx}$ & -0.01 & $81 \mathrm{xx}$ & 0.30 \\
$11 \mathrm{xx}$ & -1.16 & $38 \mathrm{xx}$ & 0.28 & $57 \mathrm{xx}$ & 0.03 & $82 \mathrm{xx}$ & 0.45 \\
$12 \mathrm{xx}$ & -1.25 & $39 \mathrm{xx}$ & 0.20 & $60 \mathrm{xx}$ & -0.74 & $83 \mathrm{xx}$ & 0.46 \\
$20 \mathrm{xx}$ & -0.71 & $40 \mathrm{xx}$ & 0.78 & $61 \mathrm{xx}$ & -1.23 & $84 \mathrm{xx}$ & 0.18 \\
$21 \mathrm{xx}$ & -0.93 & $41 \mathrm{xx}$ & 0.46 & $62 \mathrm{xx}$ & 0.90 & $85 \mathrm{xx}$ & 0.51 \\
$22 \mathrm{xx}$ & -0.40 & $42 \mathrm{xx}$ & -0.02 & $63 \mathrm{xx}$ & 0.35 & $86 \mathrm{xx}$ & 0.10 \\
$23 \mathrm{xx}$ & 0.24 & $43 \mathrm{xx}$ & 0.16 & $64 \mathrm{xx}$ & -0.35 & $87 \mathrm{xx}$ & 0.22 \\
$24 \mathrm{xx}$ & -0.14 & $44 \mathrm{xx}$ & 0.16 & $65 \mathrm{xx}$ & -0.82 & $88 \mathrm{xx}$ & 0.60 \\
$25 \mathrm{xx}$ & 0.37 & $45 \mathrm{xx}$ & 0.24 & $66 \mathrm{xx}$ & 0.43 & $89 \mathrm{xx}$ & 1.31 \\
$26 \mathrm{xx}$ & 0.43 & $46 \mathrm{xx}$ & 0.23 & $67 \mathrm{xx}$ & -0.56 & $90 \mathrm{xx}$ & 0.03 \\
$27 \mathrm{xx}$ & -0.26 & $47 \mathrm{xx}$ & 0.85 & $68 \mathrm{xx}$ & 0.01 & $91 \mathrm{xx}$ & 0.14 \\
$28 \mathrm{xx}$ & -0.12 & $48 \mathrm{xx}$ & 0.97 & $69 \mathrm{xx}$ & -0.36 & $92 \mathrm{xx}$ & 0.91 \\
$30 \mathrm{xx}$ & -0.41 & $49 \mathrm{xx}$ & 1.46 & $70 \mathrm{xx}$ & -0.67 & $93 \mathrm{xx}$ & 0.19 \\
$31 \mathrm{xx}$ & -0.51 & $50 \mathrm{xx}$ & -0.40 & $71 \times \mathrm{xx}$ & -0.45 & $94 \mathrm{xx}$ & 0.12 \\
$32 \mathrm{xx}$ & -0.22 & $51 \mathrm{xx}$ & 0.10 & $72 \mathrm{xx}$ & -1.13 & $95 \mathrm{xx}$ & -0.11 \\
$33 \mathrm{xx}$ & 0.36 & $52 \mathrm{xx}$ & 0.54 & $73 \times \mathrm{xx}$ & 0.13 & $96 \mathrm{xx}$ & 0.04 \\
$34 \mathrm{xx}$ & -0.51 & $53 \mathrm{xx}$ & 0.18 & $74 \mathrm{xx}$ & -0.40 & $97 \mathrm{xx}$ & -0.50 \\
$35 \mathrm{xx}$ & -0.50 & $54 \mathrm{xx}$ & 0.62 & $75 \mathrm{xx}$ & 0.04 & $98 \mathrm{xx}$ & 0.52 \\
$36 \mathrm{xx}$ & -0.11 & $55 \mathrm{xx}$ & -0.38 & $80 \mathrm{xx}$ & 0.58 & $99 \mathrm{xx}$ & 0.56 \\
\hline
\end{tabular}

Note: $\mathrm{PC}=$ postcode; $I_{j}^{G}=$ region-specific factor of the intercept.

region), we used a corresponding weighting, leading to the Maximum Likelihood estimates $\hat{v}=1.36$ and $\hat{\beta}=1.83$.

Since we also have to allow for having no claim in a region despite some wind speed, we determine for intervals of $10 \mathrm{~km} / \mathrm{h}$ the frequency of regions without claim and linearly interpolate these points. This results in an estimate of the probability $p(w)$ that for a given wind index $w$ there is no claim: $p(w)=(0.3508-0.0035 \cdot w) I_{\{w<95\}}$, where

$$
I_{\{w<95\}}=\left\{\begin{array}{ll}
1 & w<95 \\
0 & w \geqslant 95
\end{array} .\right.
$$



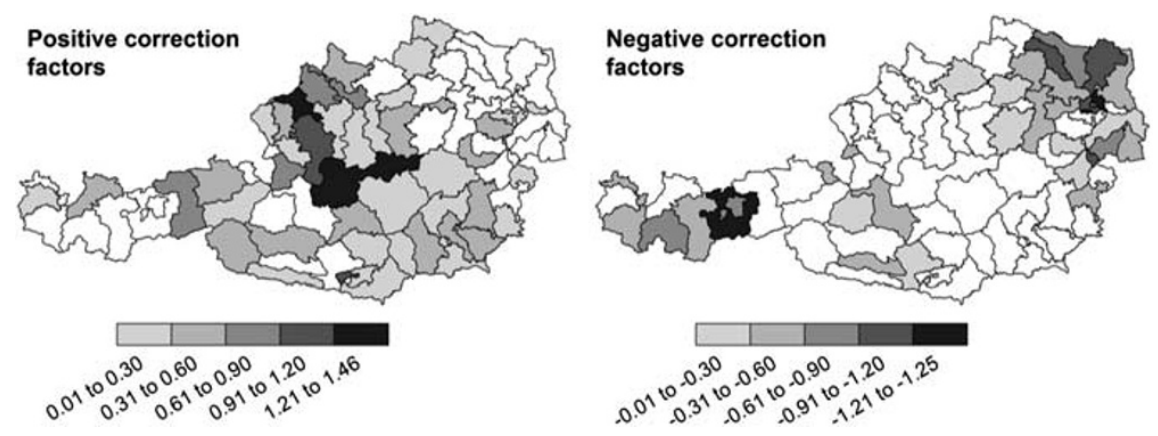

Figure 9. Correction factors $\left(I_{j}^{G}\right)$ for each two-digit postcode region (the darker, the higher is - for a given wind speed - the deviation of the expected claim in the region from the average).
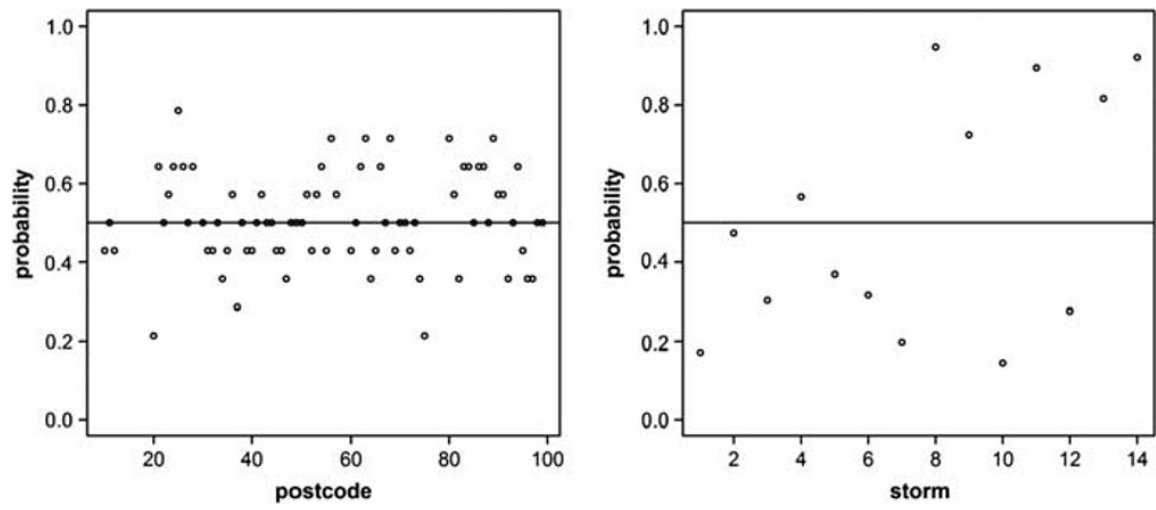

Figure 10. Relative frequency of the event 'winter storm damage larger than the model median' (left: per region, right: per storm).

Figure 10 shows the relative frequency of regional winter storm damages to be larger than the model median, both across regions and across storms. The fact that there are roughly half of the points above the model median in both cases may be interpreted as giving additional confidence in the model choice. In addition, Figure 11 shows the probability plot for the aggregate claim distribution with all data points, which may be interpreted to indicate that the aggregate distribution is acceptably captured by our model (one should also keep in mind that summer storms are supposed not to fit too well for reasons discussed above).

\section{Method 2}

As a second method we propose a Bayesian approach to estimate the parameters $s, I_{j}^{G}$, $\sigma_{I}^{2}, v$ and $\beta$ (choosing the redundant parameters $\mu_{I}=I^{L M}=0$ ). For simplicity, we use a hierarchical Bayesian model, including the $I_{i}^{S}$ as additional parameters. For the 


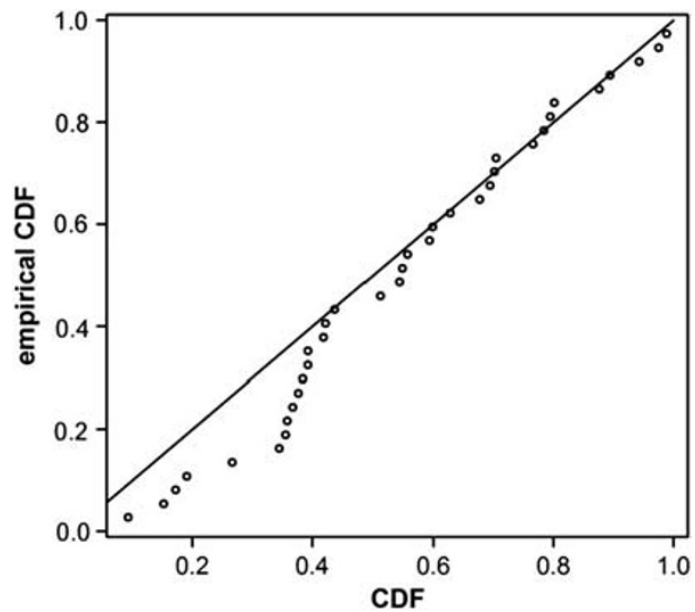

Figure 11. PP plot for the aggregate loss with all data points.

probability that there is no claim in a region we stick to the approach in Method 1 (this parameter is not essential for the final results). Now the posterior distribution of the parameters has a density proportional to:

$$
\prod_{i=1}^{n} \frac{1}{\sqrt{2 \pi \sigma_{I}^{2}}} e^{-\frac{\left(I_{i}^{S}\right)^{2}}{2 \sigma_{I}^{2}}} \prod_{j=1}^{m} \frac{\beta^{v}}{\Gamma(v)}\left|X_{i, j}-s W_{i, j}-I_{i}^{S}-I_{j}^{G}\right| e^{-\beta\left|X_{i, j}-s W_{i, j}-I_{i}^{S}-I_{j}^{G}\right|} I_{\left\{X_{i, j}>-\infty\right\}} \pi
$$

where $\pi=e^{-(\beta+v) / 10} /\left(100 \sigma_{I}^{2}\right)$ is an uninformative prior distribution, $n$ is the number of storms and $m$ the number of regions. As parameters we then take the mean of the parameters with respect to the posterior distribution. Since the means are not explicit, we have to simulate from the posterior distribution and for that purpose we use Markov Chain Monte Carlo (MCMC) ${ }^{21}$ with 320,000 simulations. For the implementation of the MCMC we use a Multiple-block Metropolis Hastings algorithm, which means that we update the parameters $s, I_{j}^{G}, I_{i}^{S}$ individually with a Metropolis Hastings algorithm, where the proposal distribution is a mixture of $\beta$ and $\gamma$ distributions that depends on the parameters. To update $\sigma_{I}^{2}$ we use a Gibbs Sampler, whereas $v$ and $\beta$ are updated similarly as in Pradhan and Kundu. ${ }^{22}$

In view of the censored data situation and the caution with which summer storm data should be treated, one could introduce weights as in Method 1, or alternatively one could reduce the data set used for calibration to the 13 winter storms that have cumulative wind index $>3,500$ and the 44 regions with the largest losses (these regions account for approximately 90 per cent of the total losses). The two approaches turn out to have similar output, so we only pursue the second method here (i.e. restrict the

\footnotetext{
${ }^{21}$ See for example Neal (1993) or Gelman et al. (2004) for an introduction.

${ }^{22}$ Pradhan and Kundu (2011).
} 


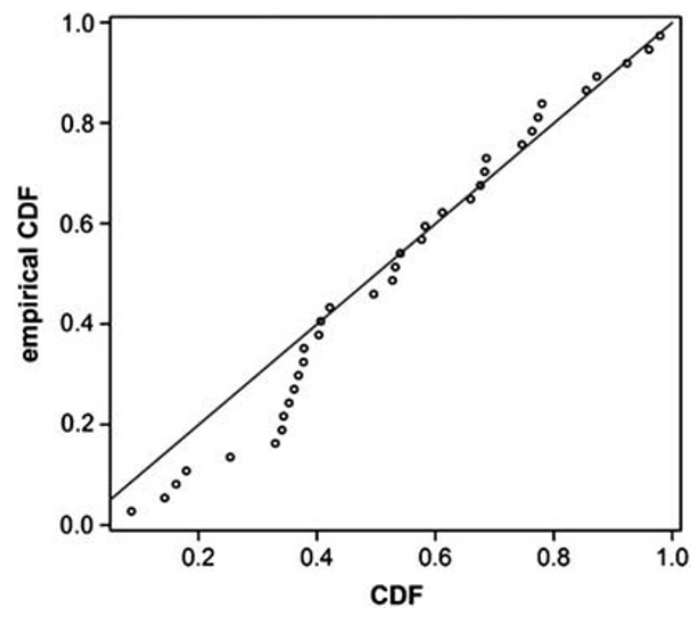

Figure 12. PP plot for the aggregate loss with all data points (model calibrated with MCMC).

calibration solely to the winter storm data in the more substantial regions). In this way one obtains $\hat{s}=0.886,(1 / n) \sum_{j=1}^{n} \hat{I}_{j}^{G}=-11.89, \hat{\sigma}_{I}^{2}=0.941, \hat{v}=0.95$ and $\hat{\beta}=1.43$. These parameters seemingly do not differ substantially from the parameters obtained by Method 1, but they make the tail of the aggregate loss distribution heavier. In Figure 12 it can be observed that the data fit looks similar to the one of Method 1 (see Figure 11). The tail difference will, however, lead to substantially different results for the quantiles.

\section{Enlarging the set of available scenarios}

A particular advantage of the above modelling approach is that in addition to the wind fields for storms in the period 1998-2009, which we used for calibration, we also have the corresponding wind fields for all storms since 1974. This naturally gives us additional scenarios of realistic wind patterns (as they have indeed appeared) and replaces the formulation of a dependence model between different regions that would be difficult to formulate (one would have to include detailed topographic information etc.) and to calibrate (in view of limited data amount). ${ }^{23}$ That is, we can calculate the building-stock-value-weighted wind index for each of these earlier storms for every region and then include those additional scenarios in a bootstrap procedure, when simulating the loss curve both on the regional and on the aggregate level. Concretely, in every simulation run we choose at random one of the (then 96) available scenarios and use our stochastic model to generate a loss output (if in such a simulation run the resulting aggregate loss is below $€ 2.5$ million, we run another loss realisation of the

${ }^{23}$ The approach of enlarging the sample of loss data for extreme value analysis by applying the developed storm model to wind data of periods, for which no historical loss data is available, has also been applied for example by Donat et al. (2011). 
Table 3 Quantiles of the aggregate loss distribution of a storm ( $€$ in million )

\begin{tabular}{lccccc}
\hline Frequency (quantile) & $20(0.95)$ & $50(0.98)$ & $100(0.99)$ & $200(0.995)$ & $250(0.996)$ \\
\hline 96 storm scenarios & 78 & 149 & 236 & 358 & 406 \\
36 storm scenarios & 68 & 124 & 182 & 258 & 287 \\
\hline
\end{tabular}

same wind scenario, in order to account for the truncation of the available data). This simple resampling method can be seen as a non-parametric bootstrap. ${ }^{24}$ Table 3 gives the resulting quantiles of such a resampling procedure for the range of interest. Note that in comparison with only 36 scenarios, the tail of the loss distribution gets considerably heavier. In other words, after normalisation, the additional historical data gives evidence that storms in Austria (on a per event basis) do not seem to have become stronger in nature (which is in line with comments of Matulla et al.). ${ }^{25}$

One may argue that a disadvantage of the above approach is that it only uses scenarios that were already realised. To improve on this, we need to extrapolate the data (in such a way, that the worst scenario has not necessarily occurred yet). Concretely, we use the cumulative wind index of a storm (i.e. the sum of the wind indices over all regions) and consider it as a random variable. In Figure 13, the full line is the best fit regression line for the storms with cumulative wind index bigger than 5,000 (intercept: 11.05 ; slope: $0.00097=0.0746 \times 77$, which is in good accordance with the slope 0.07549 of the model); the dashed lines parallel to it are at the distance to the full line of the largest residual of the storms with cumulative wind index bigger than 5,000 . Hence it seems reasonable to use the cumulative wind index for extrapolation. A moment plot of the cumulative wind indices (Figure 14) suggests that the extreme value index $\gamma=1 / \alpha$ is slightly negative. ${ }^{10}$ For simplicity, we choose here $\gamma=0$ (i.e. a distribution in the maximum domain of attraction of a Gumbel distribution), which by classical EVT entails that the excess distribution over a high threshold converges to an exponential distribution. We hence choose the 30th largest event (cumulative wind index: 5,680) as the threshold and fit an exponential distribution (with mean 592.4) to the overshoot (Figure 15 shows that the resulting fit is excellent), whereas for the values below we use the empirical distribution function. Accordingly, we now use the following variant of a bootstrap-type method to generate new wind fields: at first we randomly pick a wind field among the 96 existing ones. If the cumulative wind index is less than the threshold, we keep it; else we use the exponential distribution to get a new cumulative wind index. In order to account for the regional resolution, we proportionally scale up the wind speeds from the chosen scenario to meet the generated cumulative wind index in total. Using this method, we obtain a tail behaviour of power type with power $\alpha=1.72$ (so the resulting model is, as expected, slightly heavier tailed than the one based on the 36 scenarios, where we had $\alpha=1.83$ ). This is not as heavy as the EVT estimates of the section "EVT methodology for the

\footnotetext{
${ }^{24}$ See for example Davison (1997).

${ }^{25}$ Matulla et al. (2007).
} 


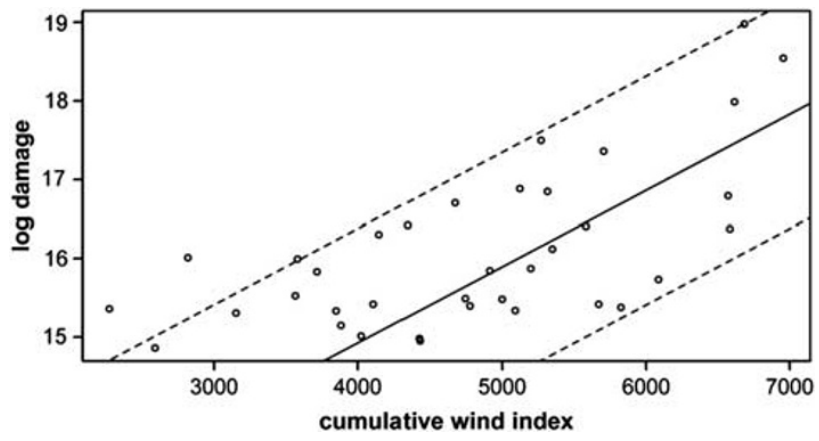

Figure 13. The cumulative wind index vs. the log aggregate damage of that storm.

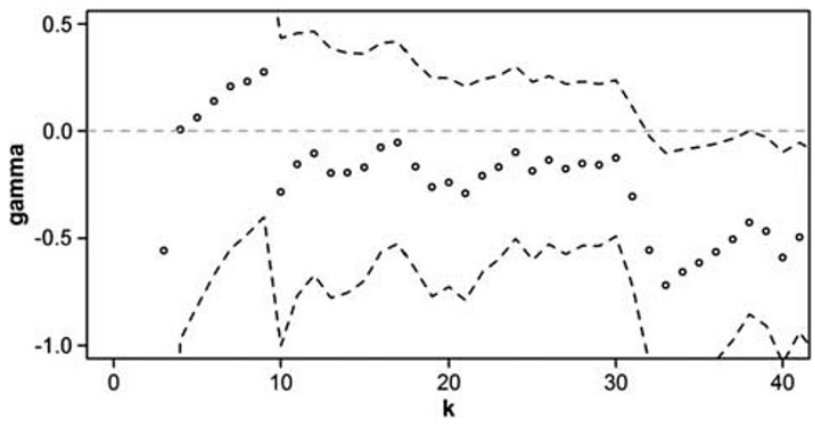

Figure 14. Moment plot for the cumulative wind index (together with the 95 per cent confidence interval).

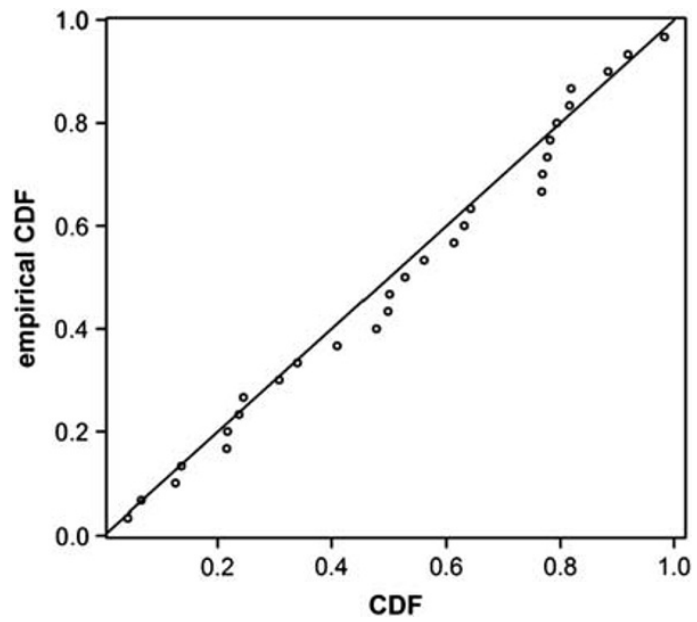

Figure 15. PP plot of the cumulative wind index above 5,680 vs. an exponential distribution. 
aggregate losses" for the value of $\alpha$, which is in line with the fact that the latter approach implicitly assumes that we have to expect worse scenarios beyond the 36 data points, whereas the additional wind scenarios (by enlarging the data set) reduce the heaviness of that "conjecture". For comparison, if we only used the 36 storms with loss data for the cumulative wind index extrapolation approach with choosing the 16th largest storm as the threshold, we would get an estimate $\alpha=1.257$ which is about the magnitude of the resulting Moment plot estimate of the section "EVT methodology for the aggregate losses" (and not far from the Hill plot estimate).

\section{The number of storms in a year}

Up to now we studied storms on a per-event basis. For certain actuarial purposes, it is also interesting to assess the distribution of the (total) annual storm loss in Austria. Recall that given our available data set from the VVO, a storm in the period 1998-2009 is registered if its inflation-adjusted induced loss exceeds €2.5 million. Figure 16 depicts the number of storms per year in this period. With 37 storm events, ${ }^{26}$ the average number of storms per year is $37 / 12 \approx 3.08$ and the empirical variance is roughly $\approx 2.265$ (here we assume that the annual losses are independent), resulting in the dispersion estimate $2.265 / 3.08=0.735$ so that the distribution seems underdispersed, which may be somewhat surprising, as one may expect over-dispersion for situations of this kind. ${ }^{27}$ However, with only 12 (annual) data points it is hard to identify any particular non-Poisson behaviour, so we decide to still fit a Poisson distribution for the number of claims with probability function:

$$
P(N=n)=\frac{\lambda^{n}}{n !} e^{-\lambda}, \quad n \geqslant 0,
$$

leading to the estimate $\hat{\lambda}=37 / 12$. As a goodness-of-fit test one can employ a $\chi^{2}$-test with classes $N \in[0,2], N \in\{3\}$ and $N \in[4, \infty)$ with each of these classes containing four realisations. The $\chi^{2}$-statistic is then 0.8417 and the $p$-value is 0.36 , but one should be cautious about statistical inference with only so few points. One may wonder whether it would be reasonable to also include the reported storm events from the period 1974-1997 (without loss data) in setting up a model for the number of claims. Figure 17 depicts the corresponding graph and it indicates an increase in the number of storms per year. Unfortunately, the definition of a storm before and after 1998 is different and so the available data situation is not sufficient to formulate a reasonably sound model that contains both periods. ${ }^{28}$ In absence of more data and information we hence decided to base the following calculations on the Poisson model discussed above and leave a more detailed study of a potential structure change in the number of

\footnotetext{
${ }^{26}$ The storm event of 23-24 July 2009 is now taken into account as well (cf. Footnote 5).

${ }^{27}$ See for example Panjer et al. (2007).

${ }^{28}$ In the set of storms before 1998 there are by far more winter storms than summer storms, whereas in the later data set with loss data there are by far more summer storms than winter storms giving further evidence that the definition of storm is likely to have changed.
} 


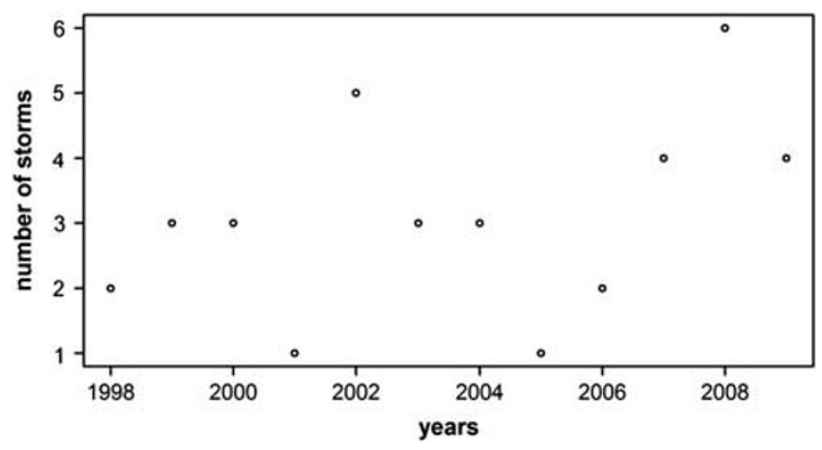

Figure 16. Number of storms with loss data, 1998-2009.

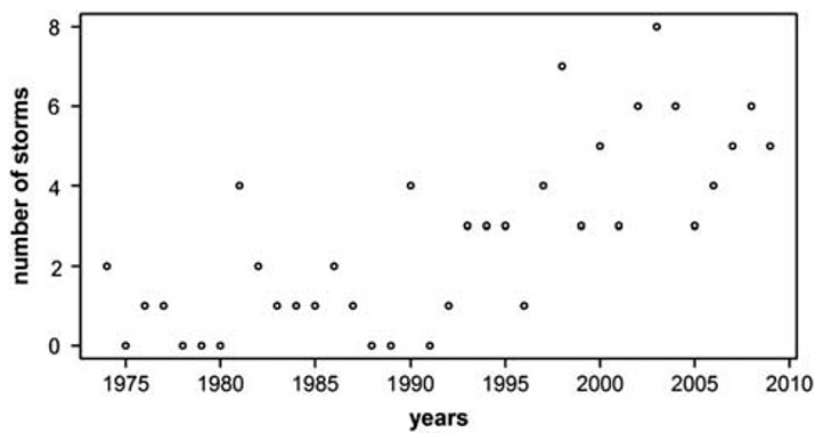

Figure 17. Number of storms with and without loss data; 1974-2009.

storm occurrences (which should be done together with climate models, etc.) for future research.

\section{Results}

We now mainly provide and discuss the results for the calibration according to Model 1, but state the corresponding results from Method 2 (MCMC calibration procedure) as well (see Tables 4 and 5). This is because we favour the former for the present situation, one reason being the more appropriate resulting tail. In particular, the resulting slope in Model 1 is in better accordance with the slope in the cumulative wind index. Using the storm loss model set up above with the extrapolated cumulative wind index and a Poisson distribution for the number of claims per year, we get an expected value for the total annual storm loss of $€ 72$ million. For the Value-at-Risk at the 99.5 per cent level, we finally obtain $€ 799$ million (see Table 4 for further quantiles). In Table 4, we also include estimated annual quantiles based on classical extreme value analysis using the asymptotic relation $\lim _{u \rightarrow \infty}\left(P\left(S_{N}>u\right)\right) /(E[N]$. $\left.P\left(X_{1}>u\right)\right)=1$ for the compound sum $S_{N}=\sum_{i=1}^{N} X_{i}$ (where $N$ denotes the random 
Table 4 Quantiles for the distribution of the annual total claim

\begin{tabular}{lcccrrr}
\hline Frequency (quantile) & $20(0.95)$ & $50(0.98)$ & $100(0.99)$ & $150(0.993)$ & $200(0.995)$ & $250(0.996)$ \\
\hline Final model & 227 & 375 & 546 & 681 & 799 & 905 \\
Model with MCMC cal. & 329 & 608 & 952 & 1,241 & 1,513 & 1,768 \\
EVT & 179 & 367 & 628 & 858 & 1,070 & 1,270 \\
Pareto-distribution & 156 & 307 & 511 & 687 & 848 & 997 \\
\hline
\end{tabular}

Note: $\mathrm{EVT}=$ Extreme Value Theory.

number of claims per year), which holds for heavy-tailed (sub-exponential) distributions of $X_{1}$ and Poisson-distributed $N^{29}$ (for the estimation of $X_{1}$, we use the moment estimator with the threshold value $k=13$ for the present purpose). These EVT quantiles should, however, be seen critically as the values result from a data set of 12 years to infer quantiles for a 200-year return period (but this approach at least improves on the alternative to first calculate the 12 empirical yearly losses and use EVT inference from those 12 points, i.e. to throw away the available intra-year information). For comparison, we also add quantiles based on a simple (plain) Pareto distribution fit for the total (inflation-adjusted) claims above $€ 2.5$ million in the third line of Table 4. The fit of the Pareto distribution is somewhat acceptable in the tail, but expectedly not so trustworthy for smaller values. As can be seen in Table 4, the 99.5 per cent quantile for the Pareto distribution is significantly smaller than the one obtained from the moment plot, which can be explained by the larger value of $\alpha$ for the former. Our stochastic model with regional solution (called "Final Model" in the table) is based on more refined information from the past, leading to a still (slightly) larger value of $\alpha$, and this may be considered the most preferable approach among the ones presented here. Accordingly, we would roughly estimate the 99.5 per cent quantile of the annual loss distribution to be $€ 800$ million. Note again that when dismissing the additional information on previous storm scenarios, this number would get closer to the one obtained by EVT techniques.

\section{Results on the level of provinces}

One particular advantage of the model approach proposed in this paper is that it is possible to assess loss distributions also for individual regions. Since in Austria the nine provinces are important sub-units for dealing with catastrophe risk also in terms of market shares of individual companies, we aggregate all two-digit postcode regions within one and the same province to get a marginal picture of the storm risk of the provinces (the two-digit postcode regions do not exactly, but sufficiently well match with the political borders of the districts). Table 5 gives for each province the actually occurred average loss and the one induced by the model. One should not expect those

\footnotetext{
${ }^{29}$ See for example Embrechts and Veraverbeke (1982) or Rolski et al. (1999). For the values in Table 4 one assumes that the asymptotic equality is exact for a given value of $u$. In this way the quantiles of the compound sum can be related to the quantiles of a single storm.
} 
Table 5 Expected losses per province

\begin{tabular}{|c|c|c|c|c|c|c|c|}
\hline & \multicolumn{2}{|c|}{ EL (empirical) } & \multicolumn{2}{|c|}{$E L(\operatorname{model} M 1)$} & \multicolumn{2}{|c|}{ EL (model M2) } & \multirow{2}{*}{$\frac{B S V}{\text { Share }(\%)}$} \\
\hline & Absolute & Share (\%) & Absolute & Share (\%) & Absolute & Share (\%) & \\
\hline "Vienna" & 3.2 & 5.6 & 5.9 & 8.2 & 8.0 & 7.3 & 18.7 \\
\hline "Lower Austria" & 12.8 & 22.4 & 17.6 & 24.3 & 27.3 & 25.0 & 21.1 \\
\hline "Upper Austria" & 22.5 & 39.3 & 31.6 & 43.7 & 41.2 & 37.8 & 15.8 \\
\hline "Salzburg" & 6.3 & 10.9 & 7.0 & 9.6 & 10.4 & 9.6 & 7.3 \\
\hline "Tyrol" & 1.2 & 2.0 & 1.5 & 2.1 & 4.1 & 3.7 & 8.2 \\
\hline "Burgenland" & 1.0 & 1.8 & 1.4 & 1.9 & 2.6 & 2.4 & 3.4 \\
\hline "Styria" & 6.7 & 11.6 & 5 & 6.9 & 10.5 & 9.6 & 14.4 \\
\hline "Carinthia" & 2.1 & 3.7 & 1.2 & 1.7 & 1.9 & 1.8 & 7.1 \\
\hline "Vorarlberg" & 1.5 & 2.6 & 1.2 & 1.6 & 3.1 & 2.9 & 4.1 \\
\hline Total & 57.2 & 100.0 & 72.4 & 100.0 & 109.1 & 100.0 & 100.0 \\
\hline
\end{tabular}

Note: $\mathrm{EL}=$ Expected Losses, $\mathrm{M} 1=$ Method 1, M2 = Method 2, BSV = Building Stock Value.

Table 6 Quantiles of the loss distribution per province (Method 1)

\begin{tabular}{lrrrrrr}
\hline Frequency (quantile) & $20(0.95)$ & $50(0.98)$ & $100(0.99)$ & $150(0.993)$ & $200(0.995)$ & $250(0.996)$ \\
\hline "Vienna" & 19 & 33 & 50 & 63 & 75 & 86 \\
"Lower Austria" & 56 & 92 & 134 & 167 & 196 & 222 \\
"Upper Austria" & 105 & 184 & 279 & 354 & 420 & 480 \\
"Salzburg" & 23 & 41 & 63 & 80 & 95 & 109 \\
"Tyrol" & 5 & 9 & 13 & 16 & 18 & 21 \\
"Burgenland" & 4 & 8 & 12 & 15 & 18 & 20 \\
"Styria" & 15 & 27 & 40 & 52 & 61 & 70 \\
"Carinthia" & 4 & 6 & 10 & 12 & 15 & 17 \\
"Vorarlberg" & 4 & 8 & 12 & 16 & 19 & 22 \\
Total & 235 & 408 & 613 & 775 & 917 & 1,047 \\
\hline
\end{tabular}

numbers to match closely, as the model also extrapolates loss potential and does not just consider what already has occurred. It may be interesting to note that the province "Upper Austria" accounts for about 40 per cent of the total claim size, yet contains only 16 per cent of the total building stock value of Austria. Table 6 depicts the resulting annual quantiles per region. In European solvency regulation rules, the 99.5 per cent quantile plays a crucial role for the capital requirement when actually insuring storm risk. One can sum up all the individual province quantiles to obtain a total of $€ 917$ million, which is to be compared with the $€ 799$ million that we obtained in the joint analysis, see Table 4 . In other words, the diversification effect (measured in terms of solvency capital relief) across provinces in Austria can be quantified to be slightly above $€ 100$ million on the 99.5 per cent quantile level, which confirms a rather strong dependence of storm risk across provinces in Austria. If the model is calibrated with MCMC (Method 2), the absolute values of the means and quantiles naturally differ, but the relative results are similar; again about 40 per cent of the total claim size are to 
Table 7 Quantiles of the loss distribution per province (Method 2)

\begin{tabular}{|c|c|c|c|c|c|c|}
\hline Frequency (quantile) & $20(0.95)$ & $50(0.98)$ & $100(0.99)$ & $150(0.993)$ & $200(0.995)$ & $250(0.996)$ \\
\hline Vienna & 24 & 46 & 76 & 102 & 126 & 148 \\
\hline Lower Austria & 83 & 154 & 246 & 321 & 390 & 458 \\
\hline Upper Austria & 126 & 250 & 411 & 549 & 674 & 789 \\
\hline Salzburg & 33 & 64 & 104 & 139 & 169 & 198 \\
\hline Tyrol & 13 & 24 & 38 & 51 & 62 & 73 \\
\hline Burgenland & 8 & 16 & 26 & 35 & 43 & 51 \\
\hline Styria & 30 & 58 & 96 & 128 & 158 & 186 \\
\hline Carinthia & 5 & 10 & 17 & 24 & 29 & 34 \\
\hline Vorarlberg & 10 & 20 & 35 & 49 & 61 & 72 \\
\hline Total & 330 & 643 & 1,050 & 1,397 & 1,711 & 2,009 \\
\hline
\end{tabular}

be expected by Upper Austria and the relative diversification effect is of the same magnitude (see Table 7).

\section{Conclusion}

We have quantitatively analysed storm damage risk for Austria, using a data set of insurance losses on a regional basis for storms over a 12-year period, using wind fields over 36 years and using building stock development in Austria in this period. We analysed this risk both on an aggregate and on a local level and set up a stochastic model that relates a suitably weighted wind speed index to the resulting damage sizes. The calibration and implementation of the model led to concrete estimates for quantiles of the resulting loss distribution, which we compared to aggregate results of classical extreme value techniques. Our findings indicate that the expected value of the aggregate loss distribution is finite (indicating insurability of storm risk in Austria), but also put on a quantitative level the difference in loss burden across provinces in Austria. In particular, the approach allows quantifying the diversification effect across provinces in Austria, which may be particularly relevant when looking for efficient insurance solutions and policies for storm risk in Austria. One should keep in mind that with 12 years of data (which themselves are partially contaminated), one has to employ extrapolation to approximate a 200 years quantile, and the corresponding results can naturally be sensitive to slight differences in the employed calibration procedure. This is also what can be observed by comparing the results for the two different calibration procedures that were implemented in the present study. We gave some arguments for preferring one procedure over the other (in particular the accordance with the EVT techniques), but such arguments must remain on a vague level. However, as described above, some quantities and relative measures remain relatively stable and one gets a feeling for the involved magnitudes. The accuracy of the procedures can be improved, once more and cleaner data sets are available.

Finally, it is not easy to compare our results with the ones of the above mentioned QIS5 impact study, in particular because the model assumptions of the latter are not 
accessible. However, the QIS5 suggestions do not seem to be in accordance with the findings resulting from our model, as can for instance be seen from the Solvency II conform capital requirement of less than 2 per cent for Tyrol as depicted in Table 6 , which is not compatible with the strong relative loading for Tyrol depicted in Figure 1. Since the numbers suggested by the QIS5 study have a significant impact on solvency capital practice, it will be interesting to reflect further about these discrepancies.

\section{References}

Beirlant, J., Goegebeur, Y., Teugels, J. and Segers, J. (2004) Statistics of Extremes: Theory and Applications, with contributions from Daniel De Waal and Chris Ferro, Wiley Series in Probability and Statistics, Chichester: John Wiley \& Sons Ltd.

Davison, A.C. (1997) Bootstrap Methods and their Applications, Cambridge Series in Statistical and Probabilistic Mathematics, Cambridge: Cambridge University Press.

de Haan, L. and Ferreira, A. (2006) Extreme Value Theory, Springer Series in Operations Research and Financial Engineering, New York: Springer.

Directive 2009/138/EC, of the European Parliament and the Council of 25 November 2009 on the taking-up and pursuit of the business of Insurance and Reinsurance (Solvency II), Official Journal of the European Union L 335/1, 17 December 2009.

Donat, M.G., Pardowitz, T., Leckebusch, G.C., Ulbrich, U. and Burghoff, O. (2011) 'High resolution refinement of a storm loss model and estimation of return periods of loss-intensive storms over Germany', Natural Hazards and Earth System Sciences 11(10): 2821-2833.

Dorland, C., Tol, R.S.J. and Palutikof, J.P. (1999) 'Vulnerability of the Netherlands and Northwest Europe to storm damage under climate change', Climatic Change 43(3): 513-535.

Draisma, G., de Haan, L., Peng, L. and Pereira, T.T. (1999) 'A bootstrap-based method to achieve optimality in estimating the extreme-value index', Extremes 2(4): 367-404.

Embrechts, P., Klüppelberg, C. and Mikosch, T. (1997) Modelling Extremal Events for Insurance and Finance, Berlin: Springer-Verlag.

Embrechts, P. and Veraverbeke, N. (1982) 'Estimates for the probability of ruin with special emphasis on the possibility of large claims', Insurance: Mathematics and Economics 1(1): 55-72.

European Commission (2010b) 'QIS5: Parameters for non-life catastrophe risk', from https://eiopa.europa .eu/consultations/qis/quantitative-impact-study-5/technical-specifications/index.html, accessed 22 July 2011.

Gelman, A., Carlin, J.B., Stern, H., Rubin, S. and Donald, B. (2004) Bayesian Data Analysis, 2nd edn, Texts in Statistical Science Series, Boca Raton, FL: Chapman \& Hall/CRC.

Haiden, T., Kann, A., Wittmann, C., Pistotnik, G., Bica, B. and Gruber, C. (2011) 'The Integrated Nowcasting through Comprehensive Analysis (INCA) system and its validation over the Eastern Alpine region', American Meteorological Society 26(2): 166-183.

Heneka, P. and Ruck, B. (2008) 'A damage model for the assessment of storm damage to buildings', Engineering Structures 30(12): 3603-3609.

Klawa, M. and Ulbrich, U. (2003) 'A model for the estimation of storm losses and the identification of severe winter storms in Germany', Natural Hazards and Earth System Sciences 3(6): 725-732.

Lescourret, L. and Robert, C.Y. (2006) 'Extreme dependence of multivariate catastrophic losses', Scandinavian Actuarial Journal 4: 203-225.

Matulla, C., Schöner, W., Alexandersson, H., von Storch, H. and Wang, X.L. (2007) 'European storminess: Late nineteenth century to present', Climate Dynamics 31(2-3): 125-130.

Neal, R.M. (1993) Probabilistic inference using Markov Chain Monte Carlo Methods, Technical Report CRG-TR-93-1.

Panjer, H., Klugman, H. and Willmot, G. (2007) Loss Models, 3rd edn, Chichester: Wiley.

Pinto, J.G., Fröhlich, E.L., Leckebusch, G.C. and Ulbrich, U. (2007) 'Changing European storm loss potentials under modified climate conditions according to ensemble simulations of the ECHAM5/ MPI-OM1 GCM', Natural Hazards and Earth System Science 7(1): 165-175.

Pradhan, B. and Kundu, D. (2011) 'Bayes estimation and prediction of the two-parameter gamma distribution', Journal of Statistical Computation and Simulation 81(9): 1187-1198. 
Reiss, R.-D. and Thomas, M. (2007) Statistical Analysis of Extreme Values with Applications to Insurance, Finance, Hydrology and Other Fields, 3rd edn, Basel: Birkhäuser Verlag.

Rolski, T., Schmidli, H., Schmidt, V. and Teugels, J. (1999) Stochastic Processes for Insurance and Finance, Wiley Series in Probability and Statistics, Chichester: John Wiley \& Sons Ltd.

\section{About the Authors}

Franz Prettenthaler is Head of Regional Science, Risk and Resource Economics unit at Joanneum Research-POLICIES, Graz, Austria. He is Lead Scientist in many European projects on quantifying catastrophic and non-catastrophic weather risks and acts as Lecturer in insurance economics at the Graz University of Technology. His previous positions include Economics Department at the University of Graz and UFR Economics at the University of Cergy-Pontoise (F). Education: Dr.rer.soc.oec 2002 (Graz, Economics), D.E.A. 2000 (Cergy, Public Economics), Mag.rer.soc.oec. 1999 (Environmental System Sciences, major Economics), M.Litt 1998 (St.Andrews, Philosophy).

Hansjörg Albrecher is a Professor of Actuarial Science at the Faculty of Business and Economics at the University of Lausanne. He also is a Faculty Member of the Swiss Finance Institute. His research interests include the modelling, quantification and control of insurance risk and risk aggregation, particularly in the presence of dependence and economic factors. Previous positions include Radon Institute of Austrian Academy of Sciences, University of Linz, Aarhus University, K.U. Leuven and Graz University of Technology. He is currently Editor of the journal Insurance: Mathematics \& Economics.

Judith Köberl is a Scientific Assistant at the Regional Science, Risk and Resource Economics unit at Joanneum Research-POLICIES, Graz, Austria. She studied Environmental System Sciences (major Economics) at the University of Graz (graduation in 2008). Her research interests include insurance economics and the economics of weather risks.

Dominik Kortschak holds a PhD from Graz University of Technology and is currently a Postdoctoral Research Fellow at the Institut de science financière et d'assurances, Université Claude Bernard Lyon 1, France. He also acknowledges support from the Swiss National Science Foundation (Project 200021-124635/1). His research interests include the tail behaviour of dependent risks, spatial and temporal dependence modelling, extreme value theory and the assessment of natural catastrophe risk. 